\title{
Ventilator-Associated Pneumonia
}

\author{
Rajesh Pande and Vikas Maurya
}

A 50-year-old diabetic male patient was admitted to the hospital with ischemic stroke $(\mathrm{GCS}=\mathrm{E} 1 \mathrm{~V} 1 \mathrm{M} 3)$. He was put on invasive positive-pressure ventilation support. On the fourth day of ICU stay, he developed fever $\left(38.6^{\circ} \mathrm{C}\right)$, a rise in total leukocyte count (156,000, N 93\%), and heterogeneous, ill-defined shadows in the right lower zone in the chest X-ray. Chest auscultation revealed bronchial breathing in the right infra-axillary area, and the nurse reported an increase in amount and purulence of secretions requiring frequent suctioning. The patient needed $5 \mathrm{mcg} / \mathrm{min}$ noradrenaline to maintain systolic blood pressure of more than $100 \mathrm{mmHg}$.

When a patient on ventilatory support develops a new or progressive infiltrate in the chest X-ray along with fever and leukocytosis after $48 \mathrm{~h}$ of intubation, it is suggestive of ventilator-associated pneumonia (VAP). This occurs in 9-27\% of intubated patients and the risk increase with the duration of mechanical ventilation. Despite a protocolized antibiotic use, the clinical cure rates are about $60 \%$ with a high recurrence rate. This can be attributed partly to the prevalence of infection with MDR pathogens like Enterobacter, Staphylococcus aureus, Klebseilla pneumoniae, Acinetobacter baumannii, Pseudomonas aeruginosa and Enterobacteriaceae (ESKAPE bugs).

\section{Step 1: Initiate Resuscitation If Patient Is in Shock}

The patient should be resuscitated, as mentioned in Chap. 23, Vol. 2.

\footnotetext{
R. Pande $(\bowtie)$

Department of Critical Care, BLK Superspeciality Hospital, New Delhi, India

V. Maurya

Department of Respiratory Medicine \& Intervention Pulmonology, Fortis Hospital, Shalimarbagh, New Delhi, India
} 


\section{Step 2: Rule Out Noninfectious Cause of Chest Infiltrate}

Various non infectious conditions can result in pulmonary shadows similar to ventilator associated pneumonia, and should be ruled out:

- Atelectasis: It is common in postoperative period following upper abdominal surgery due to hypoventilation. Left lower lobe atelectasis is common following coronary artery bypass grafting. Radiological signs include displaced fissures; crowded bronchovascular markings and shifts in the positions of the hila, diaphragm, and mediastinum; and increased density or radiopacity of the lung tissue. Fever and leukocytosis may not always be present.

- Aspiration: It occurs in patients with impaired consciousness, regurgitation of enteral feed and microaspiration around endotracheal tube cuff. Right lower lobe is commonly involved. Infiltrates may take up to $12 \mathrm{~h}$ to appear in the chest X-ray after the event. History of vomiting or bile-colored endotracheal secretions on suctioning is a clue to the diagnosis. It may be a purely chemical pneumonitis initially, caused by acidic gastric contents.

- Pulmonary embolism with infarction: It is generally acute onset in the background of risk factors for deep venous thrombosis and pulmonary embolism (PE). Diagnosis can be confirmed by CT Pulmonary Angiography.

- Pulmonary hemorrhage may resemble dense alveolar consolidation. Generally, endotracheal (ET) secretions are bloodstained. Coagulation profiles or other immunological markers may be deranged.

- Cardiogenic pulmonary edema.

- Acute respiratory distress syndrome (ARDS).

- Fluid overload.

- Hypersensitivity pneumonitis.

- Drug reactions: Cyclophosphamide, Methotrexate.

- Cryptogenic organizing pneumonia (history of recent viral infection).

- Radiation pneumonitis.

- Lung contusion.

\section{Step 3: Make a Clinical Diagnosis of VAP}

- The diagnosis of VAP is difficult, therefore scoring systems such as Clinical pulmonary infection score (CPIS) (Table 13.1) and biomarkers like $\mathrm{C}$ reactive protein (CRP) \& procalcitonin are often used in establishing the diagnosis albeit with low predictive values.

- The newly proposed ventilator-associated events (VAE) surveillance definitions (Table 13.2), endorsed by the Centre for Disease Control and Prevention should be used for epidemiological purposes only and not for diagnosing VAP.

- The new 2016 IDSA-ATS-VAP management guidelines recommend that only clinical criteria should be used for the diagnosis of VAP and scores like CPIS or markers like CRP, procalcitonin should not be used in conjunction with clinical criteria. 
Table 13.1 The modified CPIS

\begin{tabular}{l|l|l|l} 
CPIS points & 0 & 1 & 2 \\
\hline Tracheal secretions & Rare & Abundant & Abundant + purulent \\
Chest X-ray infiltrates & No infiltrate & Diffused & Localized \\
\hline Temperature $\left({ }^{\circ} \mathrm{C}\right)$ & $\geq 36.5$ and $\leq 38.4$ & $\geq 38.5$ and $\leq 38.9$ & $\geq 39$ or $\leq 36$ \\
\hline $\begin{array}{l}\text { Leukocytes count } \\
\left(\text { per } \mathrm{mm}^{3}\right)\end{array}$ & $\geq 4000$ and & $<4000$ or & $<4000$ or $>11,000+$ band \\
$\mathrm{PaO}_{2} / \mathrm{FiO}_{2}(\mathrm{~mm} \mathrm{Hg})$ & $\leq 11,000$ & $>11,000$ & forms $\geq 500$ \\
\hline Microbiology & $>240$ or ARDS & & $\leq 240$ and no evidence of \\
& Negative & & ARDS
\end{tabular}

Table 13.2 The ventilator associated events (VAE)

\begin{tabular}{|c|c|}
\hline $\begin{array}{l}\text { Ventilator associated } \\
\text { complication (VAC) }\end{array}$ & $\begin{array}{l}\text { At least one of the following indicators of worsening oxygenation } \\
\text { following a sustained period of stability or improvement on the ventilator } \\
\text { for } \geq 2 \text { calendar days } \\
\text { 1. Minimal daily } \mathrm{FiO}_{2} \text { values increase } \geq 0.20 \text { ( } 20 \text { points) over baseline \& } \\
\text { remain at or above that increased level for } \geq 2 \text { calendar days } \\
\text { 2. Minimum daily PEEP values increase } \geq 3 \mathrm{~cm} \mathrm{H}_{2} \mathrm{O} \text { over baseline or } \\
\text { above that increased level for } \geq 2 \text { calendar days }\end{array}$ \\
\hline Infection related & Patient has VAC and also fits both of the two following criteria: \\
\hline Ventilator ass & 1. Temperature greater than $38 \mathrm{C}$ \\
\hline & 2. or $\mathrm{WBC}>12,000$ or $<4000 / \mathrm{mm}^{3}$. \\
\hline (IVAC) & $\begin{array}{l}\text { 3. A new antimicrobial agent is started and is continued for } 4 \text { or more } \\
\text { calendar days. }\end{array}$ \\
\hline Possible VAP & $\begin{array}{l}\text { Patients with IVAC and one of the following criteria is met: } \\
\text { 1. Gram stain evidence of purulent respiratory secretions } \\
\text { 2. Positive respiratory culture }\end{array}$ \\
\hline Probable VAP & $\begin{array}{l}\text { Patients with IVAC and Gram stain evidence of Purulent respiratory } \\
\text { secretions plus quantitative or semiqualitative growth of a pathogenic } \\
\text { organism beyond specified thresholds for ET aspirate: } 10^{6} \text {, } \\
\text { Bronchoscopic or Mini BAL } 10^{5} \text {, PSB } 10^{3} \text {, or positive diagnostic test for } \\
\text { Legionella spp., or positive diagnostic test on respiratory secretions for } \\
\text { influenza virus, respiratory syncytial virus, adenovirus, parainfluenza } \\
\text { virus }\end{array}$ \\
\hline
\end{tabular}

The modified CPIS at baseline is calculated from the first five variables. For positive Gram stain and culture, two points are added to the CPIS baseline score. A score of more than six at baseline or after incorporating the Gram stain or culture result is considered suggestive of pneumonia. CPIS has low sensitivity and specificity for diagnosing VAP.

\section{Step 4: Send Appropriate Cultures}

- While initial resuscitation is going on, the cornerstone of therapy in suspected infection is prompt and empiric antibiotic therapy. 
- Ideally, one should send blood and endotracheal aspirate or do fiberoptic bronchoscopy with bronchoalveolar lavage for Gram stain and quantitative aerobic culture prior to starting or changing antibiotics.

- Both non-invasive (Endotracheal aspiration) sampling with Gram Stain and quantitative or semi-quantitative (Light, moderate or heavy growth) culture and invasive (mini BAL, bronchoscopic BAL or protected specimen brush) sampling with Gram stain and quantitative culture has been proposed by various guidelines for the diagnosis of VAP.

- However, if the patient is on empiric antibiotics for VAP, and invasive quantitative cultures have been done prior to starting or changing antibiotic but the results are below diagnostic threshold, then the antibiotics should be stopped.

- If there is delay in obtaining samples for logistic reasons beyond an hour, antibiotics should be given without delay.

\section{Step 5: Start Empirical Antibiotics}

1. The initial choice of antibiotics is of utmost importance. An inappropriate initial choice increases mortality.

(a) Selection of empiric antibiotic therapy should be based on the following:

- Patient risk factors for infection with MDR pathogens

The classical distinction and treatment of ventilator associated pneumonia based on early and late onset VAP concept seems inappropriate as it may result in low intensity treatment for multi-drug resistant pathogens that are commonly seen in early onset VAP as well as aggressive high intensity treatment in late onset VAP on the assumption that it is always caused by resistant pathogens. No significant difference in pathogens have been found between early \& late onset VAP. New guidelines suggest that the antibiotic therapy should focus on presence of risk factors for MDR pathogens rather than early or late VAP. It is suggested that hospital admission rather than intubation should be taken as the starting point.

(b) Recent exposure to specific antibiotic classes

(c) Local epidemiology of infection and antibiogram- the common organisms to cause VAP include Gram negative ESBL enterobacteriacae, Non fermenters like pseudomonas and acinetobacter and gram positives like MRSA

(d) Previous antibiotic therapy

2. Detailed history should be taken to identify patients who are at high risk of drugresistant infection as mentioned below:

(a) Intravenous Antimicrobial therapy in preceding 90 days

(b) Current hospitalization of 5 days or more

(c) Septic shock at the time of VAP

(d) ARDS preceding VAP

(e) Need of renal replacement therapy before VAP

3. Risk factor for MDR gram negative Bacilli and Pseudomonas aeuginosa

(a) Treatment in an ICU where more than $10 \%$ of gram negative isolates are resistant to an agent considered for monotherapy

(b) Treatment in an ICU in which local antimicrobial susceptibility rates are not known 


\section{Risk factors for MRSA}

(a) Treatment in a unit in which $>10$ to $20 \%$ of staphylococcal aureus isolates are methicillin resistant

(b) Treatment in a unit in which prevalence of MRSA is not known

\section{Step 6: Principles of Choosing an Antibiotic: (Fig. 13.1)}

1. The 2016 IDSA/ATS guidelines recommend that the choice of empiric antibiotics should be based on local distribution of pathogens and the local antibiograms.

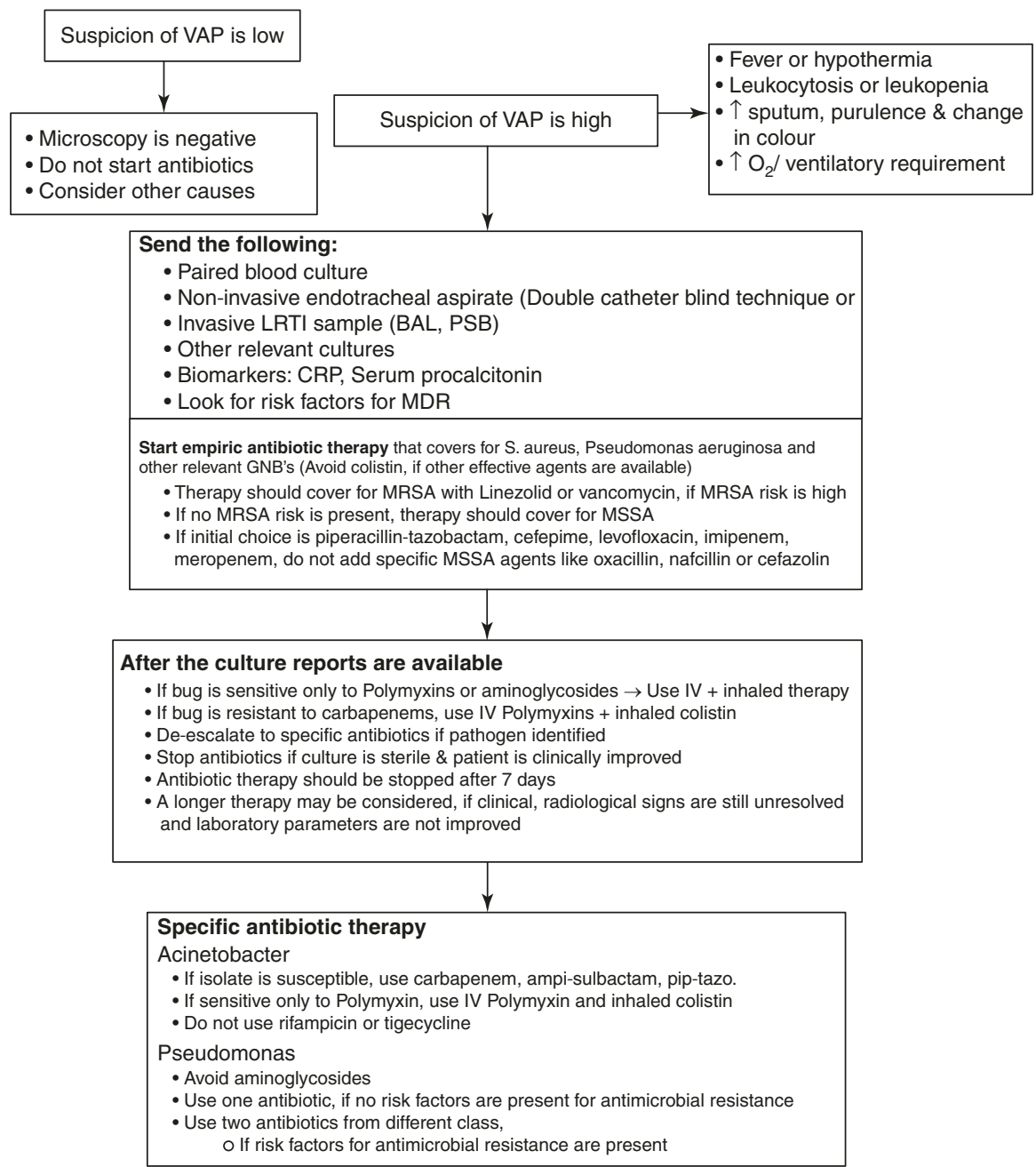

Fig. 13.1 Management of ventilator associated pneumonia 
2. Antibiotic should cover resistant gram negative organisms like ESBL in patients at risk.

3. MRSA cover should be included only if risk factors for MRSA are present, otherwise MSSA cover should be provided.

4. Avoid the antibiotic class to which the patient has been recently exposed.

5. Use parenteral antibiotics.

6. Use antibiotics in adequate dose and frequency. It is suggested to use PK/PD data for antibiotic dosing.

7. Ventilator associated tracheobronchitis should preferably not be treated with antibiotic therapy.

8. Follow the antibiotic policy of your unit/hospital.

9. For starting antibiotic therapy in suspected VAP, clinical criteria alone should be used rather than clinical criteria + serum procalcitonin or CRP or CPIS.

10. The duration of antibiotic therapy should be seven days and not more.

11. Monitoring serial procalcitonin level can help guide the decision to discontinue antibiotic

12. Specific recommendations based on new guidelines:

(a) Patients with risk factors for infection with MDR VAP

- PiperacillinTazobactam/Imipenem/Meropenem/Ceftazidime/Cefepime/ Aztreonam

Plus

- Amikacin/Gentamicin/Tobramycin/Levofloxacin/Ciprofloxacin/ Aztreonam (if not used earlier)

Plus

- Vancomycin/Linezolid/Teicoplanin

(b) Patient with No risk factor for MDR pathogen and No Risk factors for MDR gram negative pathogen and no Risk factor for MRSA

- Piperacillin/Tazobactam/Cefepime/Levofloxacin

(c) Patients with Risk factors for MRSA:

- Vancomycin/Linezolid/Teicoplanin

(d) Other recommendations

- If Piperacillin-tazobactam, cefepime, levofloxacin, imipenem or meropenem are being used for empiric cover, it is not necessary to add specific MSSA cover like oxacillin, nafcillin or cefazolin.

- Piperacillin/Tazobactam used with Vancomycin has been associated with acute kidney injury.

- Aminoglycosides, Polymyxins should be avoided in suspected VAP, if other gram negative antibiotics are available and provide adequate cover.

- In cases of VAP due to gram negative bacilli susceptible only to aminoglycosides or Polymyxins, it is suggested to use both inhaled and intravenous (systemic) antibiotics rather than systemic antibiotics alone.

- In cases of VAP due to Acinetobacter species, use a carbapenem or a BL-BLI (betalactam-beta lactam inhibitor) combination like ampicillin/sulbactam, piperacillin-tazobactam (if the isolate is susceptible to these antibiotics). 
- In cases of VAP due to Acinetobacter species sensitive only to Polymyxins, it is recommended to use intravenous Polymyxins (colistin or Polymyxin B) along with inhaled colistin. Do not use rifampicin in such cases.

- Do not use tigecycline in VAP caused by Acinetobacter species.

- Use of Inhaled antibiotics: For treating VAP, the lung concentration of the antibiotic must exceed the MIC of infecting pathogen Penetration of some systemic antibiotics like Colistin, Beta lactams, aminoglycosides and glycopeptides into lung is not as effective as flouroquinolones. Targeting lungs with aerosolized antibiotics in ventilated patients in addition to intravenous antibiotics is a good strategy and may facilitate shorter durations of therapy for multi-drug-resistant pathogens. The particle size should be $1-5 \mathrm{~mm}$ for the antibiotic to be delivered to the lower airways and the lung parenchyma.

- However, lack of specific formulation, need of special aerosolization devices and suboptimal aerosol delivery may impose limitation on the usefulness. Currently vibrating mesh nebulizers can be used for delivery of nebulized antibiotics through the ventilator circuit. Currently approved inhaled antibiotics include colistin, and tobramycin.

- Aerosolized antibiotic therapy should be synchronized to be delivered with the inspiratory flow rather than continuous nebulization. The ventilator frequency should be low to give sufficient inspiratory time for drug delivery. Humidification may be important for the patient but its use may greatly reduce the nebulized drug delivery.

\section{Step 7: Send Further Investigations}

While initial resuscitation and empirical antibiotics are being given, basic diagnostic workup should be sent. These should include the following:

- Complete blood count

- Procalcitonin, $\mathrm{C}$ reactive protein

- urea, creatinine

- Liver function test

- Prothrombin time, Na, K

- Blood culture-two sets if not sent earlier

- Endotracheal aspirates or fiberbronchoscopy with protected specimen brush (PSB) or bronchoalveolar lavage (BAL) (in selected cases like immunosuppressed)

- Arterial blood gas, lactate

- Urine for microscopy

- Chest X-ray

- ECG or echocardiogram (optional) if the patient is in septic shock 


\section{Step 8: De-escalate Antibiotics}

- Clinical improvement usually takes 2-3 days, and therapy should not be changed during this time unless the condition deteriorates. The guidelines recommend De-escalation of antibiotic therapy. Clinical criteria along with serum procalcitonin should be used for De-escalation rather than clinical criteria alone.

- If protected specimen brush (PSB) or bronchoalveolar lavage (BAL) culture results are negative in a patient who is clinically improving and hemodynamically stable, antibiotic therapy can be discontinued.

- If the culture is negative for MRSA, linezolid or vancomycin can be safely stopped, and if the culture is positive for MRSA, other antibiotics can be stopped.

- If no organism is found, one should try to look for other causes of lung shadows, that is, atelectasis, collapse, aspiration, pulmonary embolism, and hemorrhage. It decreases unnecessary exposure to antibiotics and helps to reduce resistance to antibiotics.

- Change to oral therapy once the patient accepts orally and is hemodynamically stable.

- The initial antibiotics should be given intravenously with changeover to oral therapy in responsive patients with an intact gastrointestinal function. The organism should be sensitive to oral antibiotics.

- Fluoroquinolones and linezolid are equally bioavailable in either intravenous or oral preparations.

- All patients with VAP should receive antibiotics at least for 7 days. The clinical, radiological and laboratory improvement can suggest a longer need of antibiotic therapy. Nonfermenters like acinetobacter and pseudomonas should also be treated for 14 days.

- Antibiotic dosing should be adjusted in patients with impaired renal or hepatic function.

\section{Step 9: Prevention of VAP in the ICU}

Evidence-based guidelines have recommended a number of measures that may affect the development of VAP. VAP bundles is a group of proven preventive strategies that can significantly reduce VAP rates. (Table 13.3). 
Table 13.3 Prevention of VAP

\begin{tabular}{|c|c|}
\hline Pharmacological methods & Non-pharmacological methods \\
\hline $\begin{array}{l}\text { 1. Hand hygiene with alcohol } \\
\text { based solution }\end{array}$ & 1. Use of noninvasive mask ventilation \\
\hline 2. Oral care with chlorhexidine & 2. Avoid reintubation \\
\hline $\begin{array}{l}\text { 3. Short course of antibiotic } \\
\text { therapy (when clinically } \\
\text { applicable) }\end{array}$ & 3. Orotracheal and orogastric intubation \\
\hline $\begin{array}{l}\text { 4. Sedation control and } \\
\text { weaning protocol } \\
\text { Manage without sedation } \\
\text { whenever possible/Interupt } \\
\text { sedation daily }\end{array}$ & 4. Use of heat moisture exchanger \\
\hline 5. Restricted blood transfusion & 5. Closed endotracheal suction \\
\hline $\begin{array}{l}\text { 6. Vaccines (influenza and } \\
\text { pneumococcal) } \\
\text { 7. Prophylactic probiotic }\end{array}$ & $\begin{array}{l}\text { 6. Subglottic secretion drainage } \\
\text { 7. Automated control of endotracheal tube cuff pressure } \\
\text { 8. Mechanical tooth brushing } \\
\text { 9. Ultrathin polyurethane endotracheal tube cuff } \\
\text { 10. Saline installation before endotracheal suctioning } \\
\text { 11. Change of ventilator circuit only for each new patient } \\
\text { 12. Semirecumbent positioning (elevate head end of bed } \\
30-45^{\circ} \text { ) } \\
\text { 13. Shortening duration of mechanical ventilation (assess } \\
\text { readiness to extubate daily/perform spontaneous breathing } \\
\text { trials with sedatives turned off/facilitate early mobility) } \\
\text { 14. Adequate intensive care staffing } \\
\text { 15. Use of protocol bundles } \\
\text { 16. Education and training }\end{array}$ \\
\hline
\end{tabular}

\section{Suggested Readings}

American Thoracic Society; Infectious Diseases Society of America. Guidelines for the management of adults with hospital-acquired, ventilator-associated pneumonia. Am J Respir Crit Care Med. 2005;171:388-416. ATS and IDSA have updated their VAP guidelines, in view of changing pathology of VAP and available new data on this subject.

Centers for Disease Control and Prevention. Device-associated events. Ventilator-associated event (VAE) for use in adult patients $\geq 18$ years. http://www.cdc.gov/nhsn/PDFs/pscManual/10VAE_FINAL.pdf.

Collard HR, Saint S, Matthay MA. Prevention of ventilator-associated pneumonia: an evidencebased systematic review. Ann Intern Med. 2003;138:494-50.

Dodek P, Keenan S, Cook D, Heyland D, Jacka M, Hand L, Muscedere J, Foster D, et al. Evidencebased clinical practice guidelines for the prevention of ventilator-associated pneumonia. Ann Intern Med. 2004;141:305-13. These are evidence-based practice guidelines for prevention of VAP.

Kalil AC, Metersky ML, Klompas M, et al. Management of adults with hospital-acquired and ventilator associated pneumonia: 2016 clinical practice guidelines by the Infectious Disease Society of America and the American Thoracic Society. Clin Infect Dis. 2016;63(5):e61-e111. 
Kollef MH, Morrow LE, Niederman MS, Leeper KV, Anzueto A, Benz-Scott L, Rodino FJ. Clinical characteristics and treatment patterns among patients with ventilator associated pneumonia. Chest. 2006;129:1210-8. The overall mortality rate from VAP remains unacceptably high. The de-escalation of therapy in VAP patients appears to be associated with a reduction in mortality, which is an association that warrants further clinical study.

Morrow LE, Kollef MH. Recognition and prevention of nosocomial pneumonia in the intensive care unit and infection control in mechanical ventilation. Crit Care Med. 2010;38:352-62. This article provides a brief overview of the current approaches for the diagnosis of nosocomial pneumonia and focus on strategies for prevention.

Valencia M, Torres A. Ventilator associated pneumonia. Curr Opin Crit Care. 2009;15:30-4. 